\title{
Multiterminal Conductance of a Floquet Topological Insulator
}

\author{
L. E. F. Foa Torres, ${ }^{1, *}$ P. M. Perez-Piskunow, ${ }^{1}$ C. A. Balseiro, ${ }^{2,3}$ and Gonzalo Usaj ${ }^{2,3}$ \\ ${ }^{1}$ Instituto de Física Enrique Gaviola (CONICET) and FaMAF, Universidad Nacional de Córdoba, 5000 Córdoba, Argentina \\ ${ }^{2}$ Centro Atómico Bariloche and Instituto Balseiro, Comisión Nacional de Energía Atómica, 8400 Bariloche, Argentina \\ ${ }^{3}$ Consejo Nacional de Investigaciones Científicas y Técnicas (CONICET), Argentina
}

(Received 28 August 2014; published 22 December 2014)

\begin{abstract}
We report on simulations of the dc conductance and quantum Hall response of a Floquet topological insulator using Floquet scattering theory. Our results reveal that laser-induced edge states lead to quantum Hall plateaus once imperfect matching with the nonilluminated leads is lessened. The magnitude of the Hall plateaus, however, is not directly related to the number and chirality of all the edge states at a given energy, as usual. Instead, the plateaus are dominated by those edge states adding to the time-averaged density of states. Therefore, the dc quantum Hall conductance of a Floquet topological insulator is not directly linked to topological invariants of the full Floquet bands.
\end{abstract}

DOI: 10.1103/PhysRevLett.113.266801

Introduction.-Floquet topological insulators (FTIs) [1-3] are an incarnation of topological insulators (TIs) [4-6] where the nontrivial topological properties [1] are crafted with an external driving (e.g., a circularly polarized laser). In FTIs, the Floquet chiral edge states bridge either a native bulk gap, as in semiconductor quantum wells [3], or a gap that is also produced by the driving, as in the case of graphene [7,8]. Recently, laser-induced gaps have been probed at the surface of a three-dimensional TI [9]. The field is evolving at a fast pace [10-13] with additional facets in general quantum physics [14-16], cold atoms [17-20], and photonic crystals [21].

The search for Floquet topological states has started, and some theoretical proposals $[1,7,22-24]$ embrace a realization in broadly available materials such as graphene $[25,26]$. These states could be probed through pump-probe photoemission [24], or STM [27]. But a crucial issue remains: the connection between the Floquet quasienergy spectra and the conductance.

Indeed, one of the theoretical milestones established shortly after the discovery of the quantized Hall effect [28] is the connection between the Hall conductance and a topological invariant [29] (the Chern number). This invariant, in turn, is related to the chiral edge states through the bulk-boundary correspondence [6,30]. For FTIs, the situation is more subtle. On one hand, the nonequilibrium electronic occupations $[1,31]$ pose a difficult problem if dissipation is to be considered within the system $[31,32]$. An alternative is a setup where external driving is limited to a finite region, thus leaving well-defined occupations for the asymptotic states [33-35], which can be handled through a scattering approach $[10,22]$. But even in this case, while some authors argue that the Hall conductance will be quantized within a few percent of $2 e^{2} / h$ [22], others claim that the two-terminal dc conductance may show an anomalous suppression [36]. On the other hand, there could also be
PACS numbers: 73.43.-f, 05.60.Gg, 73.63.-b, 78.67.-n

a dc current at zero bias voltage (a pumping current), thereby complicating the expected transport response.

Here, we explicitly address dc charge transport in FTIs in a multiterminal geometry (Fig. 1). First, by using a Floquet scattering picture [33-35], we can discuss the calculation of the dc conductance and address the role of the volt meters in driven systems where a current may arise even at zero bias. Later on, we do explicit calculations for the case of illuminated graphene. Our results show that the nonlocal dc Hall conductance can reach roughly constant values, once the setup is tuned to lessen the imperfect matching between the irradiated area and the nonirradiated leads. More importantly, we find a major departure from other topological systems: the magnitude of the Hall conductance plateaus is determined only by a subset of all the Floquet chiral edge states available at a given energy, breaking (a)

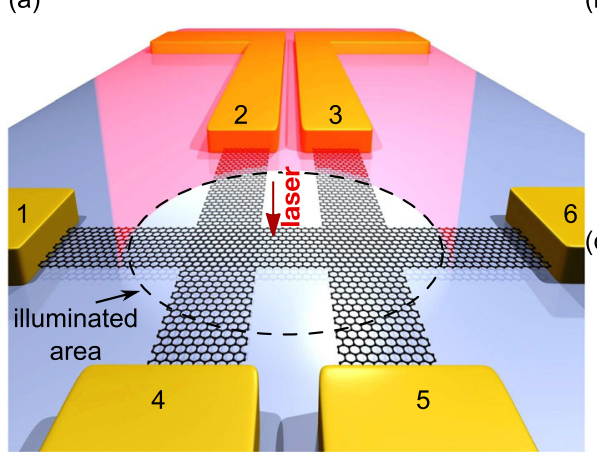

(b)

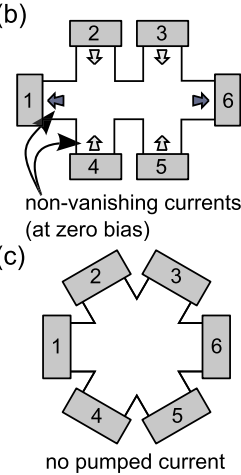

FIG. 1 (color online). (a) Scheme of a setup where a laser of frequency $\Omega$ illuminates part of a sample in a six-terminal configuration. (b) Six-terminal setup with arrows representing one of the possible directions for the currents induced at zero bias voltage (pumped currents) on each lead. (c) Six-terminal configuration in a hexagonal arrangement. For a graphene sample, this high symmetry configuration gives a vanishing pumped current. 
down the connection between topological invariants such as the winding numbers [14] and the Hall plateaus in FTIs. This result does not depend on the choice of graphene as a particular example.

Floquet theory and dc current.-Floquet theory offers a suitable framework for systems driven by a time-periodic perturbation [35,37]. One starts by noting that for a Hamiltonian $\hat{\mathcal{H}}$ with a time period $T$ there is a complete set of solutions of the form $\psi_{\alpha}(\boldsymbol{r}, t)=\exp \left(-i \varepsilon_{\alpha} t / \hbar\right) \phi_{\alpha}(\boldsymbol{r}, t)$, where $\varepsilon_{\alpha}$ is the so-called quasienergy and $\phi_{\alpha}(\boldsymbol{r}, t+T)=$ $\phi_{\alpha}(\boldsymbol{r}, t)$ is the corresponding Floquet state. By a replacement of these solutions into the time-dependent Schrödinger equation, the Floquet states turn out to satisfy a time-independent Schrödinger-like equation with the Hamiltonian being replaced by the Floquet Hamiltonian $\hat{\mathcal{H}}_{F} \equiv \hat{\mathcal{H}}-i \hbar(\partial / \partial t)$. Therefore, one has an eigenvalue problem in the direct product (Floquet) space [38], $\mathcal{R} \otimes \mathcal{T}, \mathcal{R}$ being the usual Hilbert space and $\mathcal{T}$ the space of periodic functions with period $T=2 \pi / \Omega$ (spanned by the functions $\exp (i n \Omega t)$ where the index $n$ can be assimilated to the number of "photon" excitations).

When the time-dependent potential is limited to the scattering region (either because of a finite laser spot or the screening inside metallic contacts) as in Fig. 1(a), the asymptotic states and their occupations remain well defined. The Floquet channel for incoming electrons can be set, without loss of generality, as the reference channel $n=0$; this elastic channel is naturally set apart from other replicas with $n \neq 0$, a fact that will be crucial later on. Then, one has a coherent scattering picture $[33,34]$ where dissipation is assumed to take place far in the leads. In a multiterminal setup, the time-averaged current at lead $\alpha$, $\overline{\mathcal{I}}_{\alpha}=(1 / T) \int_{0}^{T} \mathcal{I}_{\alpha}(t) d t$, is [33]

$\overline{\mathcal{I}}_{\alpha}=\frac{2 e}{h} \sum_{\beta \neq \alpha} \sum_{n} \int\left[\mathcal{T}_{\beta, \alpha}^{(n)}(\varepsilon) f_{\alpha}(\varepsilon)-\mathcal{T}_{\alpha, \beta}^{(n)}(\varepsilon) f_{\beta}(\varepsilon)\right] d \varepsilon$.

$\mathcal{T}_{\beta, \alpha}^{(n)}(\varepsilon)$ is the transmission probability for an electron from lead $\alpha$ with energy $\varepsilon$ to lead $\beta$ emitting (absorbing) $n>0$ $(n<0)$ photons, and $f_{\alpha}(\varepsilon)$ is the Fermi function at lead $\alpha$. In the absence of many-body interactions, this is equivalent to the Keldysh formalism [35,39].

The differential conductance can be obtained after a linear expansion in the bias voltage(s). Since the time-dependent potential may cause that $\sum_{\beta} \mathcal{T}_{\beta, \alpha}(\varepsilon) \neq \sum_{\beta} \mathcal{T}_{\alpha, \beta}(\varepsilon)$ $\left(\mathcal{T}_{\alpha, \beta} \equiv \sum_{n} \mathcal{T}_{\alpha, \beta}^{(n)}\right.$ is the total transmission probability), a current may appear even in the absence of a bias voltage [33].

Conductance in a two-terminal setup.-In this case $\alpha, \beta=L, R$ (left, right), and unitarity requires $\overline{\mathcal{I}}_{L}=$ $-\overline{\mathcal{I}}_{R} \equiv \overline{\mathcal{I}}$. Equation (1) can be written as

$\overline{\mathcal{I}}=\frac{2 e}{h} \int\left(\mathcal{T}(\varepsilon)\left[f_{L}(\varepsilon)-f_{R}(\varepsilon)\right]+\delta \mathcal{T}(\varepsilon)\left[f_{L}(\varepsilon)+f_{R}(\varepsilon)\right]\right) d \varepsilon$, where $\mathcal{T}(\varepsilon)=\left[\mathcal{T}_{R, L}(\varepsilon)+\mathcal{T}_{L, R}(\varepsilon)\right] / 2$ and $\delta \mathcal{T}=\left(\mathcal{T}_{R, L}-\right.$ $\left.\mathcal{T}_{L, R}\right) / 2$. We consider the zero temperature limit but generalization to finite temperature is direct. To linear order in the bias voltage $V$ we get $\overline{\mathcal{I}} \simeq\left[\left(2 e^{2}\right) / h\right] \mathcal{T}\left(\varepsilon_{F}\right) \times$ $V+\left(e^{2} / h\right) \int \delta \mathcal{T}(\varepsilon) f(\varepsilon) d \varepsilon$. The second term can be interpreted as a pumped current $\left(\mathcal{I}_{P}\right)$ resulting from the asymmetry of the transmission coefficients. Since it does not depend on the bias voltage, the differential conductance is $\left(2 e^{2} / h\right) \mathcal{T}\left(\varepsilon_{F}\right)$. In contrast to the result for timeindependent systems, the conductance depends on the transmission probabilities from left to right and from right to left. When inversion symmetry (IS) holds, both leads are indistinguishable $(\delta \mathcal{T}=0)$ and unitarity warrants zero current at zero bias. Breaking the symmetry either because of defects or slightly different contacts may introduce a large asymmetry in the transmission coefficients as we will see later on when discussing Fig. 2.

Multiterminal conductance.-In contrast to the twoterminal case, in a multiterminal setup IS does not warrant a zero pumped current (contrary to what was argued in Ref. [22]). Indeed, in a six-terminal configuration as the one in Fig. 1(b), in the absence of any bias voltage IS requires $\overline{\mathcal{I}}_{1}=\overline{\mathcal{I}}_{6}$ and $\overline{\mathcal{I}}_{2}=\overline{\mathcal{I}}_{5}$ and $\overline{\mathcal{I}}_{3}=\overline{\mathcal{I}}_{4}$, which together with charge conservation may lead to solutions where, for example, leads 2 to 5 feed nonzero currents into
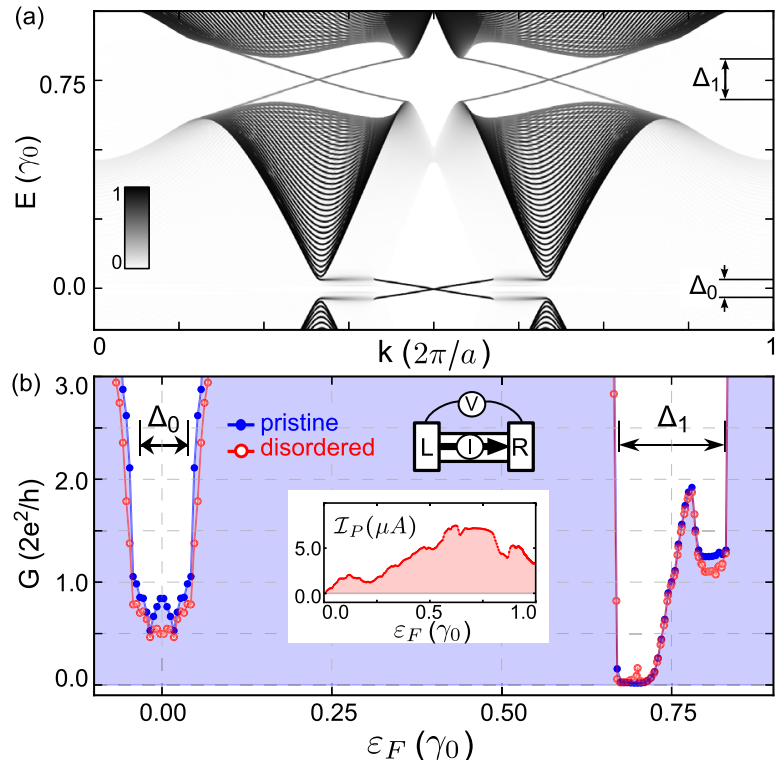

FIG. 2 (color online). (a) Quasienergy dispersion for a zigzag graphene ribbon of width $W=125 a$ illuminated by a circularly polarized laser of frequency $\hbar \Omega=1.5 \gamma_{0}$ and intensity $z=0.15$. The color scale encodes the weight of the states on the time-averaged DOS at energy $E$ : white for no weight and black for maximum weight. Replicas with $-2 \leq n \leq 2$ are considered. (b) Conductance in a two-terminal setup where a section of the infinite zigzag ribbon of length $L=420 a$ is illuminated. Full (blue) circles correspond to the pristine system while the empty (red) circles are for a ribbon with 15 vacancies distributed at random. Symmetry breaking due to defects leads to a pumped current $\mathcal{I}_{P}$ (inset). 
leads 1 and 6. Therefore, unless more stringent conditions on the setup and geometry are imposed, the volt meter may have to do additional work against the time-dependent field to keep a zero dc current.

To measure a multiterminal differential conductance, we need to establish a protocol. We start with no bias voltage and define a subset of electrodes as our volt meters [40-42]. In our discussion we assume that a volt meter is a device that adjusts its internal parameters to keep a zero dc current on the corresponding lead. In any case, the internal volt meter parameters are shifted until the associated currents [given by Eq. (1)] are zero [43]. Then one has a nonequilibrium state where we have counteracted the pumping currents at the volt meters (the source and drain may still sustain a nonzero $\mathcal{I}_{P \alpha}$ ). This state is characterized by a set of internal parameters (that we omit for brevity) and chemical potentials on each lead denoted by $\left\{\mu_{\alpha}^{(0)}\right\}$. Starting from this state and assuming that deviations $\delta \mu_{\alpha} \equiv$ $\mu_{\alpha}-\mu_{\alpha}^{(0)}$ are small enough, one gets

$$
\overline{\mathcal{I}}_{\alpha}=\mathcal{I}_{P \alpha}+\frac{2 e}{h} \sum_{\beta \neq \alpha}\left[\mathcal{T}_{\beta, \alpha}\left(\varepsilon_{F}\right) \delta \mu_{\alpha}-\mathcal{T}_{\alpha, \beta}\left(\varepsilon_{F}\right) \delta \mu_{\beta}\right],
$$

where $\mathcal{I}_{P \alpha}=0$ at the volt meters. Then we proceed as usual: fixing a small bias between two leads and imposing a zero-dc current on the volt meters. The obtained $\left\{\delta \mu_{\alpha}\right\}$ determine the conductance and Hall resistance. Hence, though it may give a remnant current at zero bias, pumping should not affect the linear conductance. When all leads are equivalent (a condition depending also on the details of the lattice), as in the setup of Fig. 1(c), the pumped currents vanish.

Conductance of irradiated graphene ribbons.Illuminating graphene with circularly polarized light can turn it into an FTI: it develops bulk band gaps [1,44] (both at the Dirac point and at $\pm \hbar \Omega / 2$ ), which are bridged by chiral edge states $[7,8]$. The two-terminal conductance of these states has only been studied at the Dirac point $[10,31]$. The nonlocal conductance in a multiterminal configuration, on the other hand, has not been explicitly examined, to the best of our knowledge, apart from a calculation based on a Kubo formula presented in Ref. [1] and an approximate analytical calculation in Ref. [22]. We address this in the following.

We consider an all-graphene system where semi-infinite graphene ribbons serve as electrodes. Graphene is modeled through a standard $\pi$-orbitals Hamiltonian $\mathcal{H}=\sum_{i} E_{i} c_{i}^{\dagger} c_{i}-$ $\sum_{\langle i, j\rangle}\left[\gamma_{i j} c_{i}^{\dagger} c_{j}+\right.$ H.c. $]$, where $c_{i}^{\dagger}$ and $c_{i}$ are the electronic creation and annihilation operators at the $\pi$ orbital localized on site $i$, which has energy $E_{i}$ ( $E_{i}$ is set equal to zero on the central area and shifted in the leads to simulate an additional doping); $\gamma_{i j}=\gamma_{0}=2.7 \mathrm{eV}$ is the nearest-neighbors hopping. The interaction with the laser (assuming normal incidence) is introduced through a time-dependent phase in the hopping amplitudes $[1,44], \gamma_{i j}=\gamma_{0} \exp \left[i\left(2 \pi / \Phi_{0}\right) \times\right.$ $\left.\int_{\boldsymbol{r}_{j}}^{r_{i}} \boldsymbol{A}(t) \cdot d \ell\right]$, where $\Phi_{0}$ is the magnetic flux quantum and $\boldsymbol{A}$ is the vector potential that is related to the electric field $\boldsymbol{E}$ through $\boldsymbol{E}=-(1 / c) \partial \boldsymbol{A} / \partial t$. The driving is assumed to take place only in the central region and is smoothly turned off before reaching the leads. $z \equiv 2 \pi a A_{0} /\left(\sqrt{3} \Phi_{0}\right)$ characterizes the laser strength, $A_{0}=|\boldsymbol{A}|$, and $a$ is the graphene lattice constant. For the numerics, we used both an implementation built on Kwant [45] and Floquet-Green's functions [44,46]. Simulating realistic laser parameters requires large systems $[7,8]$ with a high computing cost. Rather, the numerics here aim at illustrating fundamental issues of FTIs.

Figure 2(a) shows the quasienergy dispersion of a zigzag ribbon illuminated by a circularly polarized laser. The laser opens bulk gaps both at the Dirac point and at $\pm \hbar \Omega / 2$ but also produces chiral edge states bridging them. The former gap is due to the lifting of degeneracies among $n=0$ and $n= \pm 1$ replicas, while the latter is produced by a virtual process of photon emission and reabsorption [1,44]. Figure 2(b) shows the dc two-terminal conductance for both the pristine system [full (blue) circles with a dashed area] and the same ribbon with 15 random vacancies within the illuminated area [empty (red) circles]. As expected, there is a strong reduction of the conductance at the laser inducedgaps: the conductance within these bulk gaps is due to the chiral edge states. While the magnitude of the latter has the correct order of magnitude $\left(\sim 2 e^{2} / h\right)$, it presents a strong modulation as a function of energy. In addition, the presence of disorder gives a directional asymmetry: despite the small number of vacancies (less than 1\%o), there is an asymmetry in the transmission coefficients, resulting in a pumped current [Fig. 2(b) inset]. We find that this effect is even stronger in the $H$-shaped setup of Fig. 1(b) even without disorder (see the Supplemental Material [47]).

Hall conductance of irradiated graphene.-Now we turn to the Hall configuration of Fig. 1(c), where the hexagonal setup guarantees that there is no pumped current. Figures 3(a) and 3(b) show, respectively, the conductance between terminals 1 and 6 (the current source and drain, respectively) and the Hall resistance measured between 2 and 4. Empty circles with shaded area underneath are for homogeneously doped graphene $\left(E_{i}=0\right)$ whereas black (triangles) and red (solid circles) traces are for highly doped leads ( $E_{i}$ set to $0.75 \gamma_{0}$ and $1.25 \gamma_{0}$ in the leads region). Besides the strong modulation, the Hall resistance presents three main features: (i) a nonvanishing Hall response at the Floquet gaps, (ii) a Hall resistance for $\varepsilon_{F} \sim 0$ with a sign opposite that for $\varepsilon_{F} \sim \hbar \Omega / 2$, and (iii) a strong dependence of the modulation on doping. Feature (i) is produced by the Floquet chiral edge states $[1,7,8]$, while feature (ii) follows from the fact that, in this range of laser frequencies, the chiral states have opposite velocities at each gap.

The contrast between the conductance and Hall resistances just below and above the center of the dynamical gap $(E \sim \hbar \Omega / 2)$ [Figs. 3(a) and 3(b)] follows from a mismatch between states inside and outside the radiated region. Incident electrons coming from the leads belong to a well-defined Floquet channel (say $n=0$ ) and then couple 

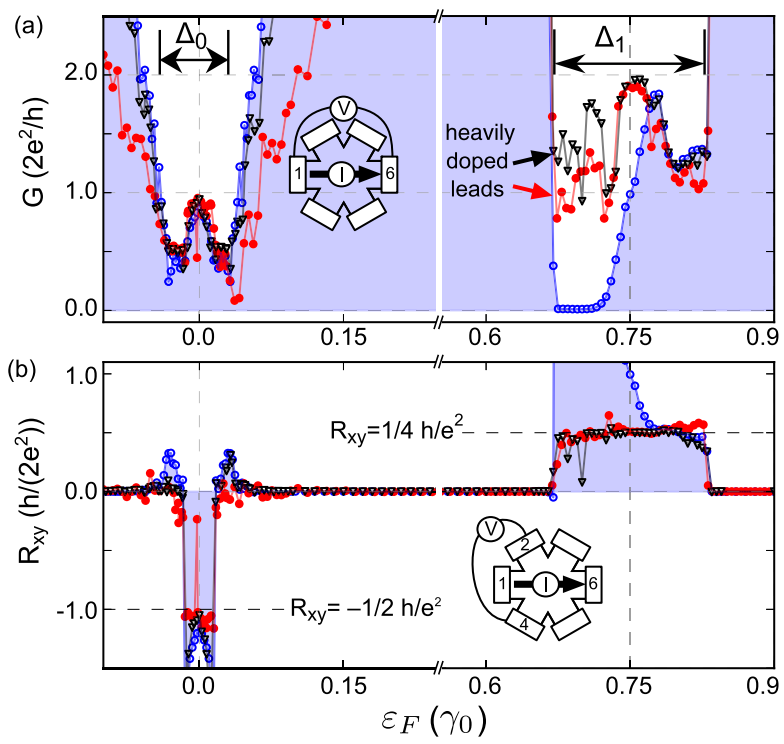

FIG. 3 (color online). Results for the setup of Fig. 1(c); parameters are as in Fig. 2(b). (a) Conductance. (b) Hall resistance (see insets); empty (blue) circles are for leads without additional doping, and (black) triangles and full circles are for $n$-doped leads (on-site energies are shifted by $0.75 \gamma_{0}$ and $1.25 \gamma_{0}$, respectively).

with the Floquet states inside the radiated region through their projection on the $n=0$ replica. Mismatch occurs because the incoming states in the leads have a projection of their pseudospin along the ribbon, which remains roughly constant across the gap while the corresponding pseudospin of the $n=0$ component of the edge state flips sign at the center of the gap, being parallel to that in the nonradiated area for $E \sim \hbar \Omega / 2^{+}$and antiparallel for $E \sim$ $\hbar \Omega / 2^{-}$[8] (Supplemental Material [47]). This gives the anomalous conductance suppression with the " $S$ " shape in Fig. 3(a). Doping the leads alleviates this mismatch since above the van Hove singularity graphene behaves as a normal metal. This is indeed what we observe in Figs. 3(a) and 3(b) (black and red lines) when the leads are heavily doped. The Hall resistance reaches roughly constant values.

In static systems, quantum Hall plateaus are determined by the number of chiral edge states, which by means of the bulk-boundary correspondence, can be related to the Chern numbers of the Bloch bands [6]. In FTIs, the Chern numbers might not be enough and other topological invariants could be needed to determine if the material is topologically trivial or not [14]. But in any case, an explicit calculation [see Figs. 4(b) and 4(c)] shows that there are indeed chiral edge states around $E \sim 0$ other than those showing up in Fig. 2(a) but that are not impacting the Hall conductance. Indeed, besides the states crossing at $k=\pi / a$ with zero energy [which are clearly distinguished in the dispersion projected on the $n=0$ channel shown in Fig. 2(a)], in Figs. 4(b) and 4(c) other chiral edge states (with opposite chirality) develop. Their projection on $n=0$ is, however, negligible because they arise from the coupling
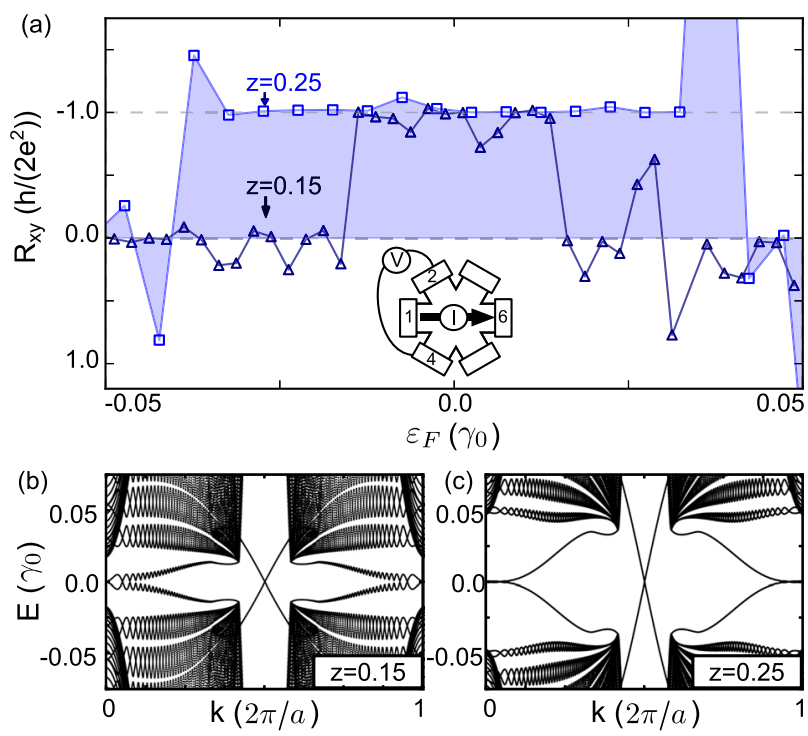

FIG. 4 (color online). (a) Detail of the Hall resistance in the hexagonal setup close to the Dirac point. Triangles (squares) are for laser intensity parametrized by $z=0.15(z=0.25)$. The results are for $\hbar \Omega=1.5 \gamma_{0}$, and zigzag terminated leads $(W=99 a, L=300 a)$ with on-site energies are shifted by $1.5 \gamma_{0}$. The full quasienergy spectra for these intensities are shown in (b) and (c). Chiral edge states other than those shown in the projection on the Floquet channel $n=0$ in Fig. 2(a) also develop but do not impact $R_{x y}$.

between the $n=-1$ and 1 replicas. Figure 4(a) shows the dc Hall resistance for small to moderate laser intensities parametrized by $z$. The leads are doped to lessen matching problems. For $z=0.25$, the plateau becomes broader, but we observe no change in its value. This shows that for Floquet topological insulators in a scattering configuration the chiral edge states do not all stand on the same footing in determining the Hall conductance plateaus.

Rather, our results support the conclusion that it is the chiral edge states with a weight on the $n=0$ channel (those adding to the time-averaged density of states, DOS, for a given energy) that determine the value of the conductance plateau (one state for $E \sim 0$ and two for $E \sim \pm \hbar \Omega / 2$ ). The reason, which does not depend on the material, is that in the weak driving regime discussed here the chiral edge states not showing up in the time-averaged DOS [encoded in the color scale in Fig. 2(a)] are very poorly coupled to the incoming electrons $(n=0$ channel). This is a major departure between FTIs and systems with time-independent Hamiltonians and one of the central results of this work.

Although the chiral edge states away from $k \sim \pi / a$ do not contribute to the Hall response, the width of the Hall plateaus in Fig. 4(a) is reduced because of photon-assisted processes involving regions with a large DOS in the higher-order bands. This is not observed at the dynamical gap, which is linear in $z$, and therefore better protected from higher-order processes. In the opposite limit of very high laser intensities, the physics may change completely since the states become highly delocalized along the Floquet channel $n$. 
Final remarks.-In summary, based on a Floquet scattering picture, we addressed the multiterminal dc conductance of driven systems. Our numerical results for the laser-induced Floquet chiral edge states in graphene show that the Hall resistance reaches plateaus when tuning the interfaces with the leads. The Hall plateaus are found not to follow the usual connection with the number or chirality of the Floquet edge states. Many of these states remain "silent," and the response is dominated by those states weighting on the time-averaged DOS. Whether these hidden edge states could manifest in other transport properties remains an open issue.

We acknowledge financial support from ANPCyT (PICTs 2006-483, 2008-2236, 2011-1552, and 20101060) and PIPs 11220080101821 and 11220110100832 from CONICET. L.E. F. F. T. and G. U. acknowledge the support of Trieste's ICTP and of the Alexander von Humboldt and Simons Foundations, respectively. Computing time from CCAD-UNC is acknowledged.

*1foa@famaf.unc.edu.ar

[1] T. Oka and H. Aoki, Phys. Rev. B 79, 081406 (2009).

[2] T. Kitagawa, E. Berg, M. Rudner, and E. Demler, Phys. Rev. B 82, 235114 (2010).

[3] N. H. Lindner, G. Refael, and V. Galitski, Nat. Phys. 7, 490 (2011).

[4] C. L. Kane and E. J. Mele, Phys. Rev. Lett. 95, 226801 (2005).

[5] M. König, S. Wiedmann, C. Brüne, A. Roth, H. Buhmann, L. W. Molenkamp, X.-L. Qi, and S.-C. Zhang, Science 318, 766 (2007).

[6] M. Z. Hasan and C. L. Kane, Rev. Mod. Phys. 82, 3045 (2010).

[7] P. M. Perez-Piskunow, G. Usaj, C. A. Balseiro, and L. E. F. Foa Torres, Phys. Rev. B 89, 121401(R) (2014).

[8] G. Usaj, P. M. Perez-Piskunow, L. E. F. Foa Torres, and C. A. Balseiro, Phys. Rev. B 90, 115423 (2014).

[9] Y. H. Wang, H. Steinberg, P. Jarillo-Herrero, and N. Gedik, Science 342, 453 (2013).

[10] Z. Gu, H. A. Fertig, D. P. Arovas, and A. Auerbach, Phys. Rev. Lett. 107, 216601 (2011).

[11] A. Gomez-Leon and G. Platero, Phys. Rev. Lett. 110, 200403 (2013).

[12] A. Kundu and B. Seradjeh, Phys. Rev. Lett. 111, 136402 (2013).

[13] M. Thakurathi, A. A. Patel, D. Sen, and A. Dutta, Phys. Rev. B 88, 155133 (2013).

[14] M. S. Rudner, N. H. Lindner, E. Berg, and M. Levin, Phys. Rev. X 3, 031005 (2013).

[15] D. Y. Ho and J. Gong, Phys. Rev. B 90, 195419 (2014).

[16] J. K. Asboth, B. Tarasinski, and P. Delplace, Phys. Rev. B 90, 125143 (2014).

[17] M. Atala, M. Aidelsburger, J. T. Barreiro, D. Abanin, T. Kitagawa, E. Demler, and I. Bloch, Nat. Phys. 9, 795 (2013).

[18] N. Goldman and J. Dalibard, Phys. Rev. X 4, 031027 (2014).
[19] S. Choudhury and E. J. Mueller, Phys. Rev. A 90, 013621 (2014).

[20] G. Jotzu, M. Messer, R. Desbuquois, M. Lebrat, T. Uehlinger, D. Greif, and T. Esslinger, Nature (London) 515, 237 (2014).

[21] M. C. Rechtsman, J. M. Zeuner, Y. Plotnik, Y. Lumer, D. Podolsky, F. Dreisow, S. Nolte, M. Segev, and A. Szameit, Nature (London) 496, 196 (2013).

[22] T. Kitagawa, T. Oka, A. Brataas, L. Fu, and E. Demler, Phys. Rev. B 84, 235108 (2011).

[23] E. Suárez Morell and L. E. F. Foa Torres, Phys. Rev. B 86, 125449 (2012).

[24] M. A. Sentef, M. Claassen, A. F. Kemper, B. Moritz, T. Oka, J. K. Freericks, and T. P. Devereaux, arXiv:1401.5103.

[25] K. S. Novoselov, A. K. Geim, S. V. Morozov, D. Jiang, M. I. Katsnelson, I. V. Grigorieva, S. V. Dubonos, and A. A. Firsov, Nature (London) 438, 197 (2005).

[26] A. H. Castro Neto, F. Guinea, N. M. R. Peres, K. S. Novoselov, and A. K. Geim, Rev. Mod. Phys. 81, 109 (2009).

[27] B. M. Fregoso, J. P. Dahlhaus, and J. E. Moore, Phys. Rev. B 90, 155127 (2014).

[28] K. von Klitzing, G. Dorda, and M. Pepper, Phys. Rev. Lett. 45, 494 (1980).

[29] D. J. Thouless, M. Kohmoto, M. P. Nightingale, and M. den Nijs, Phys. Rev. Lett. 49, 405 (1982).

[30] B. A. Bernevig and T. L. Hughes, Topological Insulators and Topological Superconductors (Princeton University Press, Princeton, NJ, 2013).

[31] H. Dehghani, T. Oka, and A. Mitra, Phys. Rev. B 90, 195429 (2014).

[32] D. W. Hone, R. Ketzmerick, and W. Kohn, Phys. Rev. E 79, 051129 (2009).

[33] M. Moskalets and M. Büttiker, Phys. Rev. B 66, 205320 (2002).

[34] S. Camalet, J. Lehmann, S. Kohler, and P. Hänggi, Phys. Rev. Lett. 90, 210602 (2003).

[35] S. Kohler, J. Lehmann, and P. Hänggi, Phys. Rep. 406, 379 (2005).

[36] A. Kundu, H. A. Fertig, and B. Seradjeh, Phys. Rev. Lett. 113, 236803 (2014).

[37] M. Grifoni and P. Hänggi, Phys. Rep. 304, 229 (1998).

[38] H. Sambe, Phys. Rev. A 7, 2203 (1973).

[39] L. Arrachea and M. Moskalets, Phys. Rev. B 74, 245322 (2006).

[40] M. Büttiker, Phys. Rev. Lett. 57, 1761 (1986).

[41] J. L. D'Amato and H. M. Pastawski, Phys. Rev. B 41, 7411 (1990).

[42] S. Datta, Electronic Transport in Mesoscopic Systems (Cambridge University Press, Cambridge, UK, 1995).

[43] This can be done through a self-consistent procedure.

[44] H. L. Calvo, H. M. Pastawski, S. Roche, and L. E. F. Foa Torres, Appl. Phys. Lett. 98, 232103 (2011).

[45] C. W. Groth, M. Wimmer, A. R. Akhmerov, and X. Waintal, New J. Phys. 16, 063065 (2014).

[46] L. E. F. Foa Torres, S. Roche, and J. C. Charlier, Introduction to Graphene-Based Nanomaterials: From Electronic Structure to Quantum Transport (Cambridge University Press, Cambridge, UK, 2014).

[47] See the Supplemental Material at http://link.aps.org/ supplemental/10.1103/PhysRevLett.113.266801 for more details on the numerics and supporting information. 